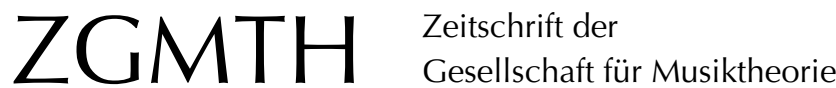

Polth, Michael (2011): In der Dachkammer. Erinnerungen an eine sagenhafte Gründung. ZGMTH 8/1, 185-187. https://doi.org/10.31751/627

\section{(C) 2011 Michael Polth}

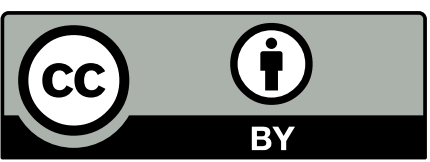

Dieser Text erscheint im Open Access und ist lizenziert unter einer Creative Commons Namensnennung 4.0 International Lizenz.

This is an open access article licensed under a

Creative Commons Attribution 4.0 International License.

veröffentlicht / first published: 14/06/2011

zuletzt geändert / last updated: 21/04/2011 


\title{
In der Dachkammer
}

\section{Erinnerungen an eine sagenhafte Gründung}

\author{
Michael Polth
}

Sehr geehrte Damen und Herren!

Zehn Jahre schon gibt es die GMTH. Anlässlich dieses Jubiläums gehen mir Bilder von damals durch den Kopf. Ich erinnere mich noch sehr genau daran, wie wir uns am 28. Juli 2000 in einem Dachkämmerchen der Hanns-Eisler-Hochschule in Berlin Mitte zur Gründungssitzung getroffen haben. Wir: Das waren Ludwig Holtmeier, Oliver SchwabFelisch, Volker Helbing, Oliver Korte, Andreas Bernnat und ich. Dieses Kämmerchen mit den schrägen Wänden war das Assistentenzimmer von Ludwig Holtmeier. Das Zimmer war schon lange der Ort unserer konspirativen Sitzungen gewesen. Vor der Gründung hatten wir uns dort über einen Zeitraum von fast anderthalb Jahren häufig, in manchen Monaten wöchentlich getroffen, um ein Konzept für eine Gesellschaft für Musiktheorie, eine Satzung und einen Internetauftritt zu entwerfen. Wenn man wie ich bei der Gründung mitgewirkt hat, wird alles, was die Gesellschaft betrifft, an den Visionen gemessen, die wir hatten - und die Gründungsvorbereitung war selbstverständlich eine Zeit der Visionen, der vorsichtigen wie der tollkühnen, der realistischen und der utopischen.

Ich erinnere mich gut, wie wir beständig versucht haben, die Strukturen der künftigen Gesellschaft zu antizipieren und die Wirkung unseres Vorhabens abzuschätzen. Wie werden die Kollegen auf die Gründung reagieren, wer wird kommen, wer wird mitmachen, mit wie vielen Mitgliedern können wir rechnen, wer wird sich verweigern? Eine Organisation der Musiktheoretiker in Deutschland war neu. Welche andere Gesellschaft sollen wir uns als Vorbild nehmen? Sollten wir uns an der Musikwissenschaft orientieren oder an der amerikanischen Musiktheorie? Was passt zu unserer Situation? Wen wollen wir erreichen? Nur die Hochschullehrer für Musiktheorie und Gehörbildung? Oder auch die Komponisten? Oder auch Musikwissenschaftler? Geht es eigentlich um eine Standesvertretung für die Theorielehrer an Musikhochschulen oder um die Sache Musiktheorie, ganz gleich wer sich damit beschäftigt?

Ein quasi inhaltliches Problem kam dazu. In den 50er und 60er Jahren des letzten Jahrhunderts herrschte das Vorurteil, Musiktheorie sei auf Satzlehre beschränkt, verfolge normative zeitlose Regeln und sei daher genuin unfähig, sich für die historische Dimension musikalischer Zusammenhänge zu öffnen. Wer nun glaubt, das sei eine schlimme Zeit für die Musiktheorie gewesen, dem sei gesagt, dass sie zur Zeit der Gründung der GMTH noch viel schlimmer gewesen ist. Wir wären froh gewesen, wenn sich die Disziplin Musiktheorie so einfach und übersichtlich dargestellt hätte, wie das vorgestrige 
Vorurteil sie sehen wollte. Tatsächlich stellten wir fest, dass alle möglichen Fachrichtungen die Musiktheorie für sich entdeckt hatten und auf ihre Art Musiktheorie betrieben. Überall tauchten musiktheoretische Reflexionen auf: bei Musikwissenschaftlern, Musikpädagogen, Mathematikern, Informatikern, Kognitionswissenschaftlern etc. Manche sagten, Musikethnologie sei eine Art von Musiktheorie, aber die Kirchenmusiker könne man auch nicht ausschließen, weil Improvisation ja implizite Theorie sei. Diese Situation hat sich bis heute eigentlich nicht geändert. Neulich wurde ich von einer Journalistin angemailt, die meine Meinung zu einer musikpsychologischen Schrift wissen wollte, von deren Autor ich noch nie etwas gehört hatte. Es stellte sich heraus, dass dieser Autor im Grunde eine - fragwürdige - Metatheorie der Funktionstheorie konzipiert hatte, die auf vermeintlichen Wahrnehmungskonstanten basierte.

Uns damals stellte diese Situation vor eine gewichtige Frage: Sollten wir alle diese Leute ansprechen und zu uns holen? Oder sollten wir uns auf sunsere beschränken? Aber was wäre diese, und aus welchem Grund diese Beschränkung?

Zerfranst war nicht nur der Gegenstand Musiktheorie, sondern auch die institutionelle Bindung. Wir kennen alle die wechselvolle Geschichte der Disziplin Musiktheorie und wissen in Deutschland um die Trennung von universitärer Musikwissenschaft und hochschulischer Musiktheorie und ihre Auswirkung auf die Bedeutung des Begriffs Musiktheorie. Es versteht sich, dass ein `Ding` wie Musiktheorie, das im Laufe seiner Geschichte inhaltlich wie institutionell zerstäubt worden ist, kaum zur Grundlage eines einfachen Konzepts von einer Gesellschaft für Musiktheorie zu machen ist.

Letztlich - so stellt es sich für mich heute dar - haben wir uns für ein Konzept der Offenheit entschieden. Wir wollten viele Gruppen ansprechen und einladen: Musiktheoretiker, ganz gleich ob sie sich als Wissenschaftler, Praktiker oder Lehrer verstehen, Musikwissenschaftler, Gehörbildungslehrer und Komponisten. Für alle sollte es Angebote zur Beteiligung geben: die Hochschulvertreterversammlung und den wissenschaftlichen Beirat, die Kongresse und die Zeitschrift. Wir sagten uns: Wir werden schon sehen, wie sich die Sache entwickelt.

Wer es Vielen recht machen will, kann auch von Vielen Prügel einstecken. Diese Erfahrung durfte ich am Anfang machen. Für eine gemeinsame Sache Musiktheorie waren durchaus nicht alle zu begeistern. Ich erinnere mich: Einem Musiktheoretiker gegenüber habe ich argumentiert, Musiktheorie werde nicht nur von Lehrern an Musikhochschulen betrieben, sie sei ein Fachgebiet, das viele verschiedene Zugänge erlaube, denen wir uns öffnen müssen. Das kam nicht gut an: »Was verstehen die denn von Musik?»

Der andere Fall: Einen hochrangigen Musikwissenschaftler hatte ich zunächst erfolgreich zur Mitarbeit im wissenschaftlichen Beitrat bewegt. Allerdings fragte er kritisch nach, warum wir überhaupt eine selbständige Gesellschaft gründen wollten. Musiktheorie gehöre schließlich zur Musikwissenschaft. Wir könnten doch innerhalb der Gesellschaft für Musikforschung einen Arbeitskreis Musiktheorie gründen. Ich entgegnete, dass die GMTH auch Musiktheoretiker von Musikhochschulen erreichen möchte, die sich nicht als Musikwissenschaftler verstehen und die nicht zur Gesellschaft für Musikforschung gehen werden. Zwei Tage nach dem Gespräch kam ein Brief des Musikwissenschaftlers mit der Absage. 
Ich denke heute: Es war richtig, eine offene Konzeption anzustreben, aber nicht, weil wir auf diese Weise alle möglichen Spielarten und Auffassungen von Musiktheorie unter unserem Dach vereinigen könnten, sondern weil die Offenheit Gelegenheit gegeben hat zu einem Prozess der Selbstfindung. Dieser Prozess ist noch nicht beendet und wird wahrscheinlich auch nicht so schnell beendet sein. Ich könnte mir aber vorstellen, dass in zehn oder zwanzig Jahren der Auseinandersetzung auf Kongressen und in der Zeitschrift sich ein einigermaßen umrissenes Gebiet der Musiktheorie herauskristallisieren könnte, das nicht die Musiktheorie in ihrer Gesamtheit repräsentieren wird, aber zumindest dasjenige, wofür man sich hier in dieser Gesellschaft zuständig fühlt.

Das Verhältnis zur Musikwissenschaft ist momentan ambivalent. Auf der Ebene der persönlichen Beziehungen gibt es viele Beispiele guter Zusammenarbeit. ^Hintenherum allerdings hört man gelegentlich von dem Vorwurf des Seperatismus. Manche - so scheint mir - verübeln uns, dass es uns als eigene Gesellschaft für Musiktheorie überhaupt gibt. Ich denke aber: Je mehr die Gesellschaft konkrete musiktheoretische Anliegen und Interessensgebiete für sich in Anspruch nimmt, umso mehr werden eben dadurch die Beziehungen zu anderen Disziplinen strukturiert. Das ambivalente Verhältnis zur Musikwissenschaft, das manche als Ressentiment der jeweils anderen Seite zu empfinden scheinen, ist wahrscheinlich die Folge unklarer Zuständigkeiten.

Als außerordentlich positiv und hoffnungsvoll empfinde ich in diesem Zusammenhang die Regelmäßigkeit der Kongresse und die damit verbundene regelmäßige Begegnung mit Kollegen. Das hat es vorher in unserem Fach so nicht gegeben. Auch war während der Gründungsvorbereitung nicht zu erwarten, dass es gelingen wird, in jedem Jahr einen Kongress an einer anderen Hochschule im deutschsprachigen Raum auszurichten. Ich glaube, dass die Institution Kongress uns bei der weiteren Entwicklung der Gesellschaft wesentlich helfen wird.

Die Kongresse zeigen aber auch etwas anderes: Durch die Gründung der GMTH wurde die deutsche Musiktheorie eigentlich nicht vereinigt. Vielmehr wurde ein Riss offensichtlich, der latent bereits bestand, ein Riss zwischen Fachvertretern, die aus ganz verschiedenen Gründen den Dialog mit Kollegen suchten, und anderen, denen daran offensichtlich wenig lag. Bis heute ist es für einen deutschen Musiktheoretiker nicht selbstverständlich, zur GTMH zu gehören und auf den Kongressen zu erscheinen, geschweige denn Bereitschaft aufzubringen, sich in einem Gremium der GMTH zu engagieren. Wenn ich mich erinnere: Wir hatten uns bei der Gründung ausgemalt, die ersten Wahlen der Gesellschaft nicht zu überleben, weil gegnerische Interessensgruppen kandidieren und sich wählen lassen werden, um die GMTH nach ihren Vorstellungen zu gestalten. Nichts dergleichen ist passiert. Bis jetzt ist der Ansturm auf die Ämter ausgeblieben.

Nach zehn Jahren mag diese Situation normal sein, gut ist sie nicht. Ich setze auf die junge Generation, für die die Existenz einer Gesellschaft der Musiktheorie Normalität ist, und wünsche mir, dass diese Leute eines Tages bei der Vorstandswahl Schlange stehen. 